Rapid Reviews COVID-19

\title{
Review 1: "The effect of eviction moratoriums on the transmission of SARS- CoV-2"
}

Published on: Feb 22, 2021

License: Creative Commons Attribution 4.0 International License (CC-BY 4.0). 


\section{$\underline{\text { RR:C19 Evidence Scale rating by reviewer: }}$}

- Potentially informative. The main claims made are not strongly justified by the methods and data, but may yield some insight. The results and conclusions of the study may resemble those from the hypothetical ideal study, but there is substantial room for doubt. Decision-makers should consider this evidence only with a thorough understanding of its weaknesses, alongside other evidence and theory. Decision-makers should not consider this actionable unless the weaknesses are clearly understood and there are other theory and evidence to further support it.

\section{Review Overview:}

The paper presents a case for a moratorium on evictions, arguing that evictions would increase the transmission of the COVID-19. The problem is exceptionally well-motivated from the recent CDC ban on evictions and court cases that challenge the CDC's arguments. The model of transmission is simple: households are modeled as a network of networks - each is a small network that has a high rate of transmission. Households are then organized into networks that allow the model to capture "doublingup" (moving to family or friends) following an eviction.

\section{Strength of evidence: Potentially Informative}

The paper studies a novel and important problem, an intervention of high public significance using powerful tools from computational epidemiology. While I have deep reservations about the conclusions, I think the paper is interesting and could spur more research.

Due to the simplified model, the model is too simplified in my view to capture the possible effects of the policy on COVID-19.

Some of the critical simplifications of the model are:

(1) Just a single parameter describes transmission outside of households. The authors do evaluate heterogeneous communities such as minority groups but do not consider other factors that affect transmission and the disease burden of COVID-19: the behaviors of individuals, employment, age, geography, and transmission through points of interest. It is probable that the model cannot realistically capture the outbreak, and indeed, there is limited model validation against real-world case data. The paper would also be stronger if real data on the eviction rate during the outbreak and could be obtained from population surveys and legal filings. 
(2) An eviction ban may have many effects on housing beyond preventing "doubling-up". Indeed, one fears unintended epidemiological effects through the reduction of the supply of housing, particularly low-cost housing. The issue is particularly vexing in my view because, while evictions affect only some $3.5 \%$ of the households, perhaps $40 \%$ of renter households move each year (in the US the average renter stays 27.5 months). Therefore, the disruption to the leasing market could have a much greater effect than considered here, and it may (a) prevent the opposite effect of doubling-up, i.e. families finding new accommodations in order to increase isolation - working adults trying to isolate themselves from elderly family members, or grown-up children moving out. (b) Homeless shelters and other group housing locations are expected to be the loci of highest transmission, but a shortage of low-cost housing would prevent individuals or even agencies from finding accommodations for residents.

\section{Readiness for publication: High}

The paper is well-organized, easy to follow, and impeccably grammatical.

I think the paper is very timely and well-written but overstates its case given the simplified model. The paper would be stronger had authors considered a more comprehensive model that includes multiple policy effects. As it is, it is a study of the doubling-up effect, not of the policy in a comprehensive way. The revised paper should address the simplifications and rebut my critique.

\section{Recommendation: Major revision}

